1 Florence Nightingale Faculty of Nursing, Midwifery, and Palliative Care, King's College, London

2 School of Health in Social Science, The University of Edinburgh

Cite this as: BMJ2022;376:0357 http://dx.doi.org/10.1136/bmi.0357

Published: 10 February 2022

\title{
A prescription for nursing: five measures to remedy the ills of the profession
}

\section{Anne Marie Rafferty and Aisha Holloway discuss five ways to improve working conditions for the nursing profession, which will also have benefits for healthcare professionals more generally}

\section{Anne Marie Rafferty, ${ }^{1}$ Aisha Holloway ${ }^{2}$}

The gaping injustices and inequalities that covid-19 has exposed globally have prompted us to try to ensure that the pandemic has at least some positive outcomes, particularly when it comes to beleaguered healthcare professionals. We set out a group of measures, which -if aligned and able to work together-we believe can put us on a strong footing to overcome some of the intractable problems that have plagued the nursing profession in the UK for decades, and which could be applied to healthcare more generally.

Pay is vital to improving recruitment and retention in nursing. It is the most tangible indicator of value and worth. Yet our pay system does not enable progression at a pace that rewards contribution, skill, and career development. The consequence of this is that our workforce is bottom heavy, with a "sticky floor"-too many colleagues are stuck in the lowest bandings. This structural weakness triggered strike action in Northern Ireland in 2019 and has consequences for the safety of patients and workplaces. Although the starting point for pay is undoubtedly important, the endpoint and career destination are too. The current situation in which only a small number of nurses progress to top leadership positions cannot provide the stability and support needed for retention, let alone meet the demands of a post-pandemic world. We have seen the toll taken on the mental and physical health and wellbeing of our colleagues during the pandemic, and the aftershocks will be with us for some time. The pay system needs re-engineering to support progression.

The second measure in our bundle is the workforce planning system, which barely exists at all, at least not in England. We desperately need a workforce planning system with a commitment to funding its outcomes, and which is aligned to a sustainable operating model for health and social care. But we only have a vague, top-down government target of 50000 nurses, with no assessment of what type of nurses are most needed and where. This is neither a planning system nor a strategy. Even if we do manage to meet the target, the government will say "job done." There is no point in decanting 50 ooo nurses into a system that continues to generate high levels of stress, burnout, and turnover in staff. ${ }^{1}$

Furthermore, the reliance on internationally recruited nurses clearly carries its own risks as was illustrated when India was overwhelmed by its own covid crisis. International recruitment can buy us some time, but we need to get our own house in order and set targets for domestic self-sufficiency. This needs to be mapped into a long term investment plan over the next 10 years.

The current supply model for the workforce of registered nurses is driven by student places and placement capacity at universities, with a few additional apprenticeships sprinkled throughout different healthcare settings. The boost to student numbers we saw with covid-19 is still not going to generate the number of nurses we need for the profession's sustained expansion to meet workforce gaps. We also need to create sustainable careers in nursing to secure a functioning leadership. Many of the chronic conditions affecting the population, let alone long covid, can be managed effectively by advanced nurse practitioners. The UK is, however, an outlier in not regulating advanced practice. It lags far behind countries such as the USA, Canada, Australia, and parts of Europe. Importantly, many countries that have regulated advanced practice do not have the levels of nursing shortages that we experience in the UK. Given the importance of advanced practice to career development we need to press for regulation as a matter of urgency. Advanced practice is an important tool for recruitment and retention of current and new cohorts of talent.

The fourth is continuing professional development (CPD). The track record of the NHS is that CPD budgets-for nurses (but not doctors) - are among the first targets when cost cutting is on the agenda. CPD needs to be demand led, based on population health needs, and modelled systematically across the career span. CPD funding is central to sustaining, retaining, and equipping the nursing workforce with the right skills to protect patient safety.

Our fifth and final measure is ensuring staffing for safe and effective care. This should be driven by data using demand modelling of population needs based on patient outcomes, with an additional allowance made for a buffer zone to build resilience and preparedness for future shocks. Better real time data on patient dependency and workflow is needed. The staffing standards recently launched by the Royal College of Nursing (RCN) are a useful starting point, but need legislative support to pinpoint who is accountable for what, as in Wales and Scotland. ${ }^{2}$

England's nursing system is beset by dysfunctionality and fragmentation, contributing to the workforce problems we see today. Many of the elements that could pave a way to improvement are already in place, but we need to align them so they can work 
together and fix the future of nursing once and for all.

Competing interests: AMR is deputy director of NIHR Health and Social Care Workforce Policy Research Unit, King's College, London. AH none declared.

Provenance and peer review: not commissioned, not peer reviewed

1 Health Committee. Workforce burnout and resilience in the NHS and social care. 2021 https://committees.parliament.uk/work/494/workforce-burnout-and-resilience-in-the-nhs-andsocial-care/

2 Royal College of Nursing. Workforce standards UK. https://www.rcn.org.uk/professional-development/publications/rcn-workforce-standards-uk-pub-009681 